\title{
Non-Motor Symptoms of the Postural Instability and Gait Difficulty Subtype in De Novo Parkinson's Disease Patients: A Cross-Sectional Study in a Single Center
}

This article was published in the following Dove Press journal:

Neuropsychiatric Disease and Treatment

\author{
Jingru Ren (iD) \\ Ping Hua' \\ Chenxi Pan' \\ Yuqian $\mathrm{Li}^{\prime}$ \\ Li Zhang ${ }^{2}$ \\ Wenbin Zhang $\mathbb{B}^{3}$ \\ Pingyi $\mathrm{Xu}^{4}$ \\ Minming Zhang ${ }^{5}$ \\ Weiguo Liu $\mathbb{D}^{\prime}$ \\ 'Department of Neurology, Affiliated Brain \\ Hospital of Nanjing Medical University, \\ Nanjing, People's Republic of China; \\ ${ }^{2}$ Department of Geriatrics, Affiliated Brain \\ Hospital of Nanjing Medical University, \\ Nanjing, People's Republic of China; \\ ${ }^{3}$ Department of Neurosurgery, Affiliated \\ Brain Hospital of Nanjing Medical \\ University, Nanjing, People's Republic of \\ China; ${ }^{4}$ Department of Neurology, First \\ Affiliated Hospital of Guangzhou Medical \\ University, Guangzhou, People's Republic of \\ China; ${ }^{5}$ Department of Radiology, Second \\ Affiliated Hospital of Zhejiang University \\ School of Medicine, Hangzhou, People's \\ Republic of China
}

Background and Purpose: Little is known about non-motor symptoms (NMSs) associated with the postural instability and gait difficulty (PIGD) phenotype, especially in de novo Parkinson's disease (PD) patients. The aims of this study were to compare NMSs between the tremor dominant (TD) and PIGD phenotypes in de novo PD patients and to determine factors that are associated with the PIGD subtype.

Patients and Methods: In a cross-sectional study conducted at our single center, 226 de novo PD patients with a median disease duration of 2 years were recruited. Data, including comprehensive demographics, motor subtypes and NMSs were obtained. Motor subtypes were classified as PIGD and non-PIGD (TD and indeterminate) by Jankovic's method. NMSs were evaluated by the non-motor symptoms questionnaire (NMSQuest).

Results: We identified 73 (32.3\%), 34 (15.0\%) and 119 (52.7\%) patients with TD, intermediate and PIGD subtypes, respectively. Patients with the PIGD subtype had poorer ADL, motor, depression, anxiety, sleep, and non-motor scores compared with those with the TD subtype. In the NMSQuest, the prevalence of cardiovascular, sleep, mood/cognitive and miscellaneous domains was increased in patients with the PIGD subtype compared with patients with the TD subtype. Multivariable forward stepwise logistic regression revealed that the Hamilton Depression Scale (HAMD) [odds ratio (OR), 1.059; 95\% confidence interval (CI), 1.016-1.104, $p=0.007]$ and pain (OR, 3.175; 95\% CI, 1.695-5.947, $p<0.001)$ exhibit significant discriminative power in differentiating PIGD and non-PIGD groups.

Conclusion: The PIGD group had more severe cardiovascular symptoms, sleep impairments, mood disturbances and pain. We demonstrated for the first time that pain was associated with the PIGD phenotype. Prompt detection and early treatment of NMSs related to the PIGD phenotype may improve patient outcomes.

Keywords: de novo Parkinson's disease, motor subtypes, non-motor symptoms, tremor dominant, postural instability and gait difficulty

\section{Introduction}

Parkinson's disease (PD) is a chronically progressive neurodegenerative disorder with a wide variety of clinical manifestations that encompass both motor and non-motor symptoms (NMSs). ${ }^{1}$ The heterogeneity in clinical manifestations of PD has been conceptualized by a taxonomy of subgroups based on family history, age at onset, variable progression, patterns in motor symptoms, and NMSs, such as dementia and
Department of Neurology, Affiliated Brain Hospital of Nanjing Medical University, 264 Guangzhou Road, Gulou District, Nanjing 210029, People's Republic of China

Tel +86-25-82296569

Email wgliunbh@sina.com 
depression. ${ }^{2-5}$ The clinical phenotypes of PD can be subdivided into postural instability and gait difficulty (PIGD) and non-PIGD (tremor dominant (TD) and indeterminate) subtypes empirically according to the presence of prominent motor symptoms. ${ }^{2}$ Compared to the non-PIGD phenotype, the PIGD phenotype is associated with more rapid disease progression and less favorable outcomes. ${ }^{2,6}$ However, the pathophysiology underlying the PIGD phenotype remains unclear.

In addition to motor disturbances, various NMSs, including sleep disorders, neuropsychiatric symptoms, and cognitive impairment, are experienced by PD patients. ${ }^{7}$ The onset of some NMSs, such as hyposmia, REM sleep behavior disorder (RBD) and constipation, may precede the diagnosis of PD by years and significantly increase the risk of developing $\mathrm{PD}$, as they are prodromal $\mathrm{PD}$ markers. ${ }^{8-10}$ In addition, some NMSs are insensitive to dopaminergic therapy, which may result from extensive nondopaminergic neurodegeneration. ${ }^{11}$ As the disease progresses, NMSs, rather than motor disability, have a major detrimental effect on patients' prognoses and quality of life. ${ }^{12,13}$ Despite their clinical significance, NMSs remain under-recognized and consequently undertreated. ${ }^{14}$

Therefore, a comprehensive understanding of NMSs related to the PIGD subtype has the potential to provide further insights into the pathogenesis, clinical progression, and personalized treatment of the PIGD subtype. Previous studies have reported that patients with the PIGD subtype exhibit a wide variety of NMSs, especially in patients with advanced PD. ${ }^{15-17}$ Nevertheless, to the best of our knowledge, there is a paucity of work on NMSs of the PIGD subtype in de novo PD patients. To address these gaps in knowledge, the goals of this study were to examine NMSs of the PIGD phenotype in de novo PD patients and to identify factors that are related to the PIGD phenotype. We hypothesized that specific NMSs are associated with the PIGD subtype.

\section{Patients and Methods}

\section{Patients}

A total of 226 idiopathic PD patients diagnosed on the basis of the United Kingdom Parkinson's Disease Society Brain Bank clinical diagnostic criteria were recruited from the Department of Neurology at the Affiliated Brain Hospital of Nanjing Medical University from January 2012 to December 2019. ${ }^{18}$ Patients were followed up at least once and exhibited positive responsiveness to levodopa. The exclusion criteria for this study were as follows: (1) individuals who were undergoing or underwent treatment with antiparkinsonian agents, (2) those with middle- or latestage PD (modified H-Y stage $\geq 3$ ), (3) those with atypical or secondary parkinsonism, (4) those with clinically significant lesions visible on brain magnetic resonance imaging (MRI) scans, (5) those with a severe chronic condition (eg, renal failure, cardiac failure, diabetes with complications), and (6) those who were unable to complete clinical evaluations.

This research was approved by the Medical Ethics Committee of Affiliated Brain Hospital of Nanjing Medical University, and the approval numbers were "2011-KY003 and 2015-KY030." This study was performed in accordance with the Declaration of Helsinki. All participants provided written informed consent prior to the experiment.

\section{Clinical Evaluation}

Demographic and clinical characteristics of the patients were recorded before dopaminergic medication was started. The participants answered questions about their age, gender, years of formal education, age at onset of PD, and disease duration in years. The participants' level of independence during activities of daily living (ADL) was assessed using ADL (part II) subscales of the Unified Parkinson's Disease Rating Scale (UPDRS). The state of parkinsonism was evaluated by the motor (part III) subscales of the UPDRS and the modified Hoehn-Yahr (H-Y) staging system. General cognition was measured with the Mini-Mental State Examination (MMSE) and Montreal Cognitive Assessment (MoCA). Mood was assessed with the Hamilton Depression Scale (HAMD) and Hamilton Anxiety Scale (HAMA). Sleep was measured with the Parkinson Disease Sleep Scale (PDSS). NMSs were evaluated by the non-motor questionnaire (NMSQuest). ${ }^{19}$ The NMSQuest consists of 30 items that are divided into nine domains (cardiovascular, sleep, mood/cognitive, perceptual problems/hallucinations, attention/memory, gastrointestinal, urinary, sexual function and miscellaneous). ${ }^{20}$

\section{Subtype Classifications}

The patients were included in either the PIGD or non-PIGD (TD and indeterminate) subtype groups based on the method proposed by Jankovic. ${ }^{2}$ The ratio of the mean UPDRS tremor scores (UPDRS III items 20-21 and UPDRS II item 16) to the mean UPDRS PIGD scores (UPDRS III items 29 and 30 and UPDRS II items 13-15) was used to identify PIGD PD patients (ratio $\leq 1$ ), TD PD patients (ratio $\geq 1.5$ ) and indeterminate PD patients (ratios $>1.0$ and $<1.5$ ). 


\section{Statistical Analysis}

IBM SPSS software version 25.0 was used to perform all statistical analyses. The Kolmogorov-Smirnov test was used to determine the normality of quantitative data. The differences in the gender distributions were evaluated using chi-square tests. Among TD/indeterminate/PIGD subtypes, one-way analysis of variance (ANOVA) was performed to compare the differences in demographic and clinical variables other than gender when both sets of data followed a normal distribution; for non-normally distributed data, the KruskalWallis H-test was used. A two-sample $t$-test was performed to compare the differences in demographic and clinical variables other than gender between the TD and PIGD groups when both sets of data followed a normal distribution; for nonnormally distributed data, the Mann-Whitney $U$-test was used. Statistical comparisons of the prevalence of NMSs between the TD and PIGD groups were performed using the chi-square test. Univariable and multivariable forward stepwise logistic regressions were performed to identify potential factors to differentiate the PIGD motor phenotype versus the non-PIGD motor phenotype. All variables with $p<0.20$ in the univariate logistic regression were included in a forward stepwise procedure to build the final multivariable model. $P<0.05$ was considered statistically significant.

\section{Results}

\section{Demographic and Clinical Characteristics}

The demographic and clinical characteristics of all 226 patients with de novo PD are summarized in Table 1. To elucidate the features of the PIGD subtype, we compared the distributions of demographic and clinical characteristics between the TD and PIGD groups. No significant differences in age, gender, years of formal education, age at onset, disease duration, H-Y stage, MMSE score or MoCA score were noted. Scores related to ADL, motor symptoms, and NMSs, including the UPDRS ADL, UPDRS motor, HAMD, HAMA, PDSS and NMSQuest scores, were significantly different between the two groups. The patients with the PIGD subtype had poorer ADL ( $p=$ $0.004)$, motor $(p=0.013)$, depression $(p<0.001)$, anxiety $(p=0.003)$, sleep $(p=0.040)$, and non-motor scores $(p=$ $0.001)$ compared with TD subtype patients (Table 1).

Table I Demographic and Clinical Characteristics of Overall Study Population

\begin{tabular}{|c|c|c|c|c|c|c|}
\hline Variables & $\begin{array}{l}\text { Overall } \\
(n=226)(100 \%)\end{array}$ & $\begin{array}{l}\text { TD } \\
(n=73)(32.3 \%)\end{array}$ & $\begin{array}{l}\text { Intermediate } \\
(n=34)(15.0 \%)\end{array}$ & $\begin{array}{l}\text { PIGD } \\
(n=I \mid 9)(52.7 \%)\end{array}$ & p-value ${ }^{a}$ & p-value ${ }^{b}$ \\
\hline Age (years) & $61.07 \pm 10.22$ & $59.48 \pm|| .3 \mid$ & $63.91 \pm 8.78$ & $61.23 \pm 9.79$ & 0.109 & 0.259 \\
\hline Gender (male) & III (49.1\%) & $39(53.4 \%)$ & $18(52.9 \%)$ & $54(45.4 \%)$ & 0.495 & 0.279 \\
\hline Formal education & $9.0(6.0,12.0)$ & $9.0(6.0,12.0)$ & II.5 (5.8, I5.0) & $9.0(6.0,12.0)$ & 0.747 & 0.723 \\
\hline Age at onset & $58.50 \pm|0.3|$ & $57.03 \pm 11.65$ & $60.79 \pm 9.18$ & $58.74 \pm 9.67$ & 0.199 & 0.273 \\
\hline Disease duration & $2.0(1.0,3.0)$ & $2.0(1.0,3.5)$ & $1.0(1.0,5.0)$ & $2.0(1.0,3.0)$ & 0.521 & 0.218 \\
\hline $\mathrm{H}-\mathrm{Y}$ stage & $1.5(1.0,2.0)$ & $1.5(1.0,2.0)$ & $1.5(1.0,2.0)$ & $1.5(1.0,2.0)$ & 0.629 & 0.560 \\
\hline UPDRS ADL & $8.0(5.0,12.0)$ & $7.0(4.0,11.0)$ & $9.5(6.0,13.5)$ & $9.0(5.0,13.0)$ & 0.011 & 0.004 \\
\hline UPDRS motor & $20.0(14.0,27.0)$ & $18.0(10.0,25.5)$ & $23.0(12.75,28.75)$ & $21.0(15.0,27.0)$ & 0.043 & 0.013 \\
\hline MMSE & $28.0(26.0,29.0)$ & $28.0(26.0,29.0)$ & $29.0(26.0,30.0)$ & $28.0(26.0,29.0)$ & 0.522 & 0.660 \\
\hline $\mathrm{MoCA}$ & $24.0(20.0,26.0)$ & $24.0(20.0,26.8)$ & $25.0(21.0,27.0)$ & $24.0(20.0,26.0)$ & 0.169 & 0.525 \\
\hline HAMD & $8.0(4.0,13.0)$ & $6.0(2.0,11.0)$ & $7.0(4.0,12.0)$ & $9.0(5.0,16.0)$ & 0.001 & $<0.001$ \\
\hline HAMA & $6.0(3.0,11.0)$ & $4.0(2.0,9.0)$ & $5.0(3.0,11.0)$ & $7.0(4.0,12.0)$ & 0.008 & 0.003 \\
\hline PDSS & $128.5(109.8,140.3)$ & $130.0(|| 4.0, \mid 43.5)$ & |36.5 (| | | .5, |44.5) & $124.0(100.0,138.0)$ & 0.030 & 0.040 \\
\hline NMSQuest & $8.0(5.0,12.0)$ & $6.0(4.0,9.0)$ & $8.0(4.8,12.3)$ & $9.0(6.0,12.0)$ & 0.004 & 0.001 \\
\hline
\end{tabular}

Notes: Data are presented as the mean \pm SD, $\mathrm{n}(\%)$ and median (interquartile range). Bold values are statistically significant ( $p<0.05)$. ${ }^{\mathrm{a}}$ Comparisons of variables among TD, PIGD, and intermediate subtypes. Univariate $p$-values calculated using ANOVA, Kruskal-Wallis $\mathrm{H}$-test, or Chi-square test. ${ }^{b}$ Comparisons of variables between TD and PIGD subtypes. Univariate $p$-values calculated using Student's $t$ test, Mann-Whitney U-test, or Chi-squared test.

Abbreviations: TD, tremor dominant; PIGD, postural instability and gait difficulty; H-Y, Hoehn and Yahr; UPDRS, Unified Parkinson's Disease Rating Scale; ADL, activities of daily living; MMSE, Mini-Mental State Examination; MoCA, Montreal Cognitive Assessment; HAMD, Hamilton Depression Scale; HAMA, Hamilton Anxiety Scale; PDSS, Parkinson Disease Sleep Scale; NMSQuest, Non-motor Questionnaire. 


\section{NMSs Between TD and PIGD Motor Subtypes}

As the total NMSQuest score significantly differed between the TD and PIGD groups, the prevalence of symptoms corresponding to each domain and item of the NMSQuest in the two groups was further compared in Table 2. "Orthostatic symptoms" in the cardiovascular domain ( $p=$ $0.003)$, "vivid dream imagery" in the sleep domain ( $p=$ $0.023)$, "low mood" and "anxiety" in the mood domain ( $p=$ $0.045, p=0.011$ ), and "pain" in the miscellaneous domain $(p=0.001)$ were more prevalent in patients with the PIGD subtype compared with patients with the TD subtype. No differences between the TD and PIGD subtypes were noted in the remaining NMSQuest items.

\section{Potential Factors of the PIGD Motor Phenotype}

With univariate logistic regression, the following candidate factors of the PIGD motor phenotype were identified: UPDRS ADL $(p=0.005)$, UPDRS motor $(p=0.013)$, HAMD $(p=0.001)$, HAMA $(p=0.008)$, PDSS $(p=0.008)$, NMSQuest $(p=0.007)$, cardiovascular domain $(p=0.021)$, sleep domain $(p=0.027)$, mood/cognitive domain $(p=0.035)$, weight change $(p=0.199)$ and pain $(p<0.001)$ (Table 3$)$. Then, with multivariable forward stepwise logistic regression, the following factors were excluded as potential risk and protective factors: the UPDRS ADL, UPDRS motor, HAMA, PDSS, NMSQuest, cardiovascular domain, sleep domain, mood/cognitive domain and weight change. The HAMD [odds ratio (OR), 1.059; 95\% confidence interval (CI), 1.016-1.104, $p=0.007$ ] and pain (OR, 3.175; 95\% CI, 1.695-5.947, $p<0.001)$ had significant discriminative power in differentiating the PIGD group from the non-PIGD group (Table 4). Patients with higher HAMD score and incidence of pain exhibited a positive correlation with the PIGD phenotype.

\section{Discussion}

Our research deepens the understanding of NMSs related to the PIGD subtype by exploring the characteristics of NMSs in the PIGD subtype and determining potential factors of the PIGD subtype in de novo PD patients. The results in previous studies evaluating NMSs of the PIGD phenotype are limited by the utilization of medications with known antidopaminergic effects, long durations from PD diagnosis to study recruitment and differences in the severity of PD in patients. ${ }^{15,16,21,22}$ In addition, it is worth noting that the
Table 2 Comparison of Non-Motor Symptoms (NMS) Between the Tremor Dominant (TD) and Postural Instability and Gait Difficulty (PIGD) Groups of Patients with De Novo Parkinson's Disease (PD)

\begin{tabular}{|c|c|c|c|}
\hline Variables & $\begin{array}{l}\text { TD }(n=73) \\
(32.3 \%)\end{array}$ & $\begin{array}{l}\text { PIGD (n=1 I9) } \\
(52.7 \%)\end{array}$ & $p$-value \\
\hline DI. Cardiovascular & $19(26.0)$ & $57(47.9)$ & 0.003 \\
\hline I. Orthostatic symptoms & $17(23.3)$ & $53(44.5)$ & 0.003 \\
\hline 2. Falls & $5(6.8)$ & $9(7.6)$ & 0.854 \\
\hline D2. Sleep & $54(74.0)$ & $102(87.7)$ & 0.043 \\
\hline 3. Daytime somnolence & $12(16.4)$ & $25(21.0)$ & 0.436 \\
\hline 4. Insomnia & $24(32.9)$ & $50(42.0)$ & 0.207 \\
\hline 5. Vivid dream imagery & $30(4 I .1)$ & $69(58.0)$ & 0.023 \\
\hline 6. Somnambulate & $20(27.4)$ & $45(37.8)$ & 0.139 \\
\hline 7. Restless legs & $20(27.4)$ & $36(30.3)$ & 0.673 \\
\hline D3. Mood/cognitive & $39(53.4)$ & $85(71.4)$ & 0.011 \\
\hline 8. Loss of interest & $25(34.2)$ & $48(40.3)$ & 0.399 \\
\hline 9. Low mood & $29(39.7)$ & $65(54.6)$ & 0.045 \\
\hline 10. Anxiety & $28(38.4)$ & $68(57.1)$ & 0.011 \\
\hline $\begin{array}{l}\text { D4. Perceptual problems/ } \\
\text { hallucinations }\end{array}$ & $24(32.9)$ & $43(36.1)$ & 0.646 \\
\hline II. Hallucinations & $3(4.1)$ & $6(5.0)$ & 1.000 \\
\hline 12. Delusions & $2(2.7)$ & $4(3.4)$ & 1.000 \\
\hline 13. Impaired taste or smell & $11(15.1)$ & $28(23.5)$ & 0.157 \\
\hline 14. Diplopia & II (15.1) & $22(18.5)$ & 0.542 \\
\hline D5. Attention/memory & $53(72.4)$ & $87(73.1)$ & 0.939 \\
\hline 15. Forgetfulness & $44(60.3)$ & $80(67.2)$ & 0.328 \\
\hline 16. Impaired attention & $29(39.7)$ & $55(46.2)$ & 0.379 \\
\hline D6. Gastrointestinal & $45(61.6)$ & $83(69.7)$ & 0.248 \\
\hline 17. Dysphagia & $14(19.2)$ & $28(23.5)$ & 0.479 \\
\hline 18. Nausea & $6(8.2)$ & $13(10.9)$ & 0.542 \\
\hline 19. Constipation & $27(37.0)$ & $60(50.4)$ & 0.069 \\
\hline 20. Bowel incontinence & $2(2.7)$ & $2(1.7)$ & 0.618 \\
\hline $\begin{array}{l}\text { 21. Incomplete bowel } \\
\text { emptying }\end{array}$ & $19(26.0)$ & $43(36.1)$ & 0.146 \\
\hline 22. Sialorrhea & $9(12.3)$ & $27(22.7)$ & 0.074 \\
\hline D7. Urinary & $29(39.7)$ & $48(40.3)$ & 0.933 \\
\hline 23. Urinary urgency & $22(30.1)$ & $33(27.7)$ & 0.720 \\
\hline 24. Nocturia & $17(23.3)$ & $28(23.5)$ & 0.969 \\
\hline D8. Sexual function & $12(16.4)$ & $21(17.6)$ & 0.829 \\
\hline 25. Impaired libido & $12(16.4)$ & $18(15.1)$ & 0.392 \\
\hline 26. Sexual dysfunction & $8(11.0)$ & $16(13.4)$ & 0.613 \\
\hline D9. Miscellaneous & $32(43.8)$ & $83(69.7)$ & $<0.001$ \\
\hline 27. Weight change & $9(12.3)$ & $27(22.7)$ & 0.074 \\
\hline 28. Pain & $15(20.5)$ & $52(43.7)$ & 0.001 \\
\hline 29. Lower limb swelling & $7(9.6)$ & $16(13.4)$ & 0.424 \\
\hline 30. Excessive sweating & $22(30.1)$ & $41(34.5)$ & 0.536 \\
\hline
\end{tabular}

Notes: Data are expressed as $\mathrm{n}(\%)$. Bold values are statistically significant $(p<0.05)$. Univariate $p$-values calculated using Chi-square test.

prevalence of PIGD in this study (52.7\%) was significantly increased compared with that of the Parkinson's Progression Biomarker Initiative (PPMI) cohort (17.5\%) and Deprenyl 
Table 3 Univariate Logistic Regression for Potential Factors of the Postural Instability and Gait Difficulty (PIGD) Phenotype in De Novo Parkinson's Disease (PD)

\begin{tabular}{|c|c|c|c|c|c|}
\hline Variables & $\boldsymbol{\beta}$ & SE & $p$-value & OR & $95 \% \mathrm{Cl}$ \\
\hline Age & 0.003 & 0.013 & 0.803 & 1.003 & $0.978-1.029$ \\
\hline Gender (male) & -0.316 & 0.267 & 0.236 & 0.729 & $0.432-|.23|$ \\
\hline Formal education & -0.002 & 0.028 & 0.938 & 0.998 & $0.945-1.053$ \\
\hline Age at onset & 0.005 & 0.013 & 0.707 & 1.005 & $0.980-1.03 \mid$ \\
\hline Disease duration & -0.009 & 0.043 & 0.838 & 0.991 & $0.910-1.079$ \\
\hline UPDRS ADL & 0.094 & 0.034 & 0.005 & 1.099 & $1.029-1.174$ \\
\hline UPDRS motor & 0.036 & 0.015 & 0.013 & 1.037 & $1.008-1.067$ \\
\hline $\mathrm{H}-\mathrm{Y}$ stage & 0.196 & 0.294 & 0.506 & 1.216 & $0.683-2.164$ \\
\hline MMSE & -0.021 & 0.034 & $0.54 I$ & 0.979 & $0.915-1.048$ \\
\hline $\mathrm{MoCA}$ & -0.026 & 0.026 & 0.312 & 0.974 & $0.926-1.025$ \\
\hline HAMD & 0.066 & 0.020 & 0.001 & 1.068 & $1.027-1.112$ \\
\hline HAMA & 0.064 & 0.024 & 0.008 & 1.066 & $1.017-1.117$ \\
\hline PDSS & -0.016 & 0.006 & 0.008 & 0.984 & $0.973-0.996$ \\
\hline NMSQuest & 0.083 & 0.031 & 0.007 & 1.086 & $1.023-1.154$ \\
\hline DI. Cardiovascular & 0.637 & 0.276 & 0.021 & 1.891 & $\mathrm{I} .10 \mathrm{I}-3.248$ \\
\hline D2. Sleep & 0.755 & 0.342 & 0.027 & 2.127 & $1.088-4.158$ \\
\hline D3. Mood/cognitive & 0.596 & 0.282 & 0.035 & 1.815 & $1.044-3.154$ \\
\hline D4. Perceptual problems/hallucinations & 0.027 & 0.278 & 0.923 & 1.027 & $0.596-1.77 \mid$ \\
\hline D5. Attention/memory & -0.086 & 0.304 & 0.777 & 0.918 & $0.506-1.664$ \\
\hline D6. Gastrointestinal & -0.061 & 0.292 & 0.833 & 0.940 & $0.531-1.667$ \\
\hline D7. Urinary & -0.109 & 0.270 & 0.686 & 0.897 & $0.528-1.523$ \\
\hline D8. Sexual function & 0.126 & 0.357 & 0.724 & 1.134 & $0.563-2.286$ \\
\hline \multicolumn{6}{|l|}{ D9. Miscellaneous } \\
\hline Weight change & 0.441 & 0.343 & 0.199 & 1.554 & $1.945-6.642$ \\
\hline Pain & 1.279 & 0.313 & $<0.001$ & 3.595 & $1.945-6.642$ \\
\hline Lower limb swelling & 0.031 & 0.393 & 0.936 & 1.032 & $0.478-2.229$ \\
\hline Excessive sweating & 0.254 & 0.287 & 0.378 & 1.289 & $0.734-2.264$ \\
\hline
\end{tabular}

Notes: Bold values are statistically significant $(p<0.20)$. P-values calculated using univariate logistic regression analysis.

Abbreviations: UPDRS, Unified Parkinson's Disease Rating Scale; ADL, activities of daily living; H-Y, Hoehn and Yahr; MMSE, Mini-Mental State Examination; MoCA, Montreal Cognitive Assessment; HAMD, Hamilton Depression Scale; HAMA, Hamilton Anxiety Scale; PDSS, Parkinson Disease Sleep Scale; NMSQuest, Non-motor Questionnaire.

Table 4 Multivariable Forward Logistic Regression for Potential Factors of the Postural Instability and Gait Difficulty (PIGD) Phenotype in De Novo Parkinson's Disease (PD)

\begin{tabular}{|l|l|l|l|l|l|}
\hline Variables & $\boldsymbol{\beta}$ & $\mathbf{S E}$ & $\boldsymbol{p}$-value & $\mathbf{O R}$ & $\mathbf{9 5 \%} \mathbf{C l}$ \\
\hline HAMD & 0.057 & 0.021 & 0.007 & 1.059 & $1.016-1.104$ \\
Pain & 1.155 & 0.320 & $<0.001$ & 3.175 & $1.695-5.947$ \\
\hline
\end{tabular}

Abbreviations: SE, standard error; OR, odds ratio; $\mathrm{Cl}$, confidence interval; HAMD, Hamilton Depression Scale.

and Tocopherol Antioxidative Therapy of Parkinsonism (DATATOP) cohort $(29.1 \%),{ }^{2,23}$ which may be partly because an increasing number of TD patients will enter the PIGD subgroup as the duration of the disease increases. ${ }^{24}$ In this study, the median disease duration of patients with de novo PD was 2 years. In comparison, based on the goals of PPMI, the study recruited very early PD patients with an average disease duration starting at 6.7 months after diagnosis. Similarly, the average disease duration of the DATATOP cohort, which is the basis of the TD/PIGD classification, was 1.0 years. $^{25}$

Norepinephrine (NE) deficiency in the central and peripheral nervous systems of PD patients correlates with the PIGD phenotype, sleep impairment, affective disorders and cardiovascular symptoms. ${ }^{26}$ Diagnosed PD patients with bradykinesia and rigidity exhibit a more extensive NE deficiency in the brain. ${ }^{27}$ Further, freezing of gait (FOG), a unique subtype of the PIGD subtype, can also be caused by a reduction in NE innervation in the brain. ${ }^{28}$ Therefore, central NE deficiency tends to be noted in the PIGD subtype rather than the TD subtype in PD patients. In addition, central NE deficiency is also related to sleep impairment and affective disorders. A reduction in $\mathrm{LC}$ and its projections to the cerebral cortex, basal forebrain, thalamus, dorsal raphe, pedunculopontine and laterodorsal tegmental nucleus cause 
sleep disorders. ${ }^{29}$ Emotions, including depression and anxiety are partly governed by NE activity. ${ }^{30}$ In addition to central NE degeneration, peripheral noradrenergic cardiac denervation is one of the earliest pathophysiological characteristics of PD and causes cardiovascular symptoms. ${ }^{26,31-33}$ PIGD subtype patients had more severe sleep impairments, mood disturbances and cardiovascular symptoms, indicating that the PIGD subtype is connected with more widespread and diffuse neurodegeneration of NE in the central and peripheral nervous systems. Therefore, treatment strategies based on dopaminergic treatment with increased NE enhancement may improve these symptoms of the PIGD subtype. A detailed understanding of NMSs related to the PIGD subtype may improve research on its etiology, subtype-specific biomarkers and therapeutic paradigms of the PIGD subtype.

HAMD and pain are associated with the PIGD subtype. As depression and pain became more severe, the proportion of patients with the PIGD subtype of PD increased. Depression can occur in patients throughout early stages of $\mathrm{PD}$, including the preclinical, prodromal and clinical stages. In preclinical PD, the occurrence of depression is an integral component of progressive neurodegeneration, which eventually leads to dopaminergic neuron death. ${ }^{34}$ In prodromal $\mathrm{PD}$, depression, an important prodromal marker, markedly increases an individual's risk of developing PD and occurs within five years before PD is clinically diagnosed. ${ }^{9,35}$ In clinical PD, depression is associated with the PIGD subtype rather than the non-PIGD subtype in early PD patients, which may be attributed to the similar pathophysiology of NE deficiency between depression and the PIGD subtype. ${ }^{26,36,37}$ Therefore, we believe that depression is an independent risk factor for the PIGD subtype. Importantly, we demonstrated for the first time that pain is related to the PIGD phenotype. To the best of our knowledge, there is no detailed report on the relationship between pain and the PIGD phenotype of PD. Pain is not only an early symptom of PD but also a premotor symptom, and the incidence of pain increases as the disease progresses. A recent systematic review and meta-analysis proposed that pain in PD patients is attributable to a dopamine deficiency leading to obvious hyperalgesia. ${ }^{38}$ Dopaminergic striatal denervation in a PIGD group of de novo PD patients was significantly increased compared with that of a nonPIGD group at baseline. ${ }^{39}$ Therefore, pain is more common in patients with the PIGD subtype compared with patients with the non-PIGD subtype. Additionally, in a previous retrospective study on self-perceived premotor symptoms and a cross-sectional study using the King's Parkinson's Disease Pain Scale (KPPS), PIGD patients exhibited an increased frequency of pain compared with non-PIGD patients. ${ }^{40,41}$ These evidences suggest that pain is connected with the PIGD subtype. Patients with depression and pain may be warned of a risk of developing the PIGD subtype.

Several caveats must be taken into consideration when interpreting our findings. First, this cross-sectional study was only applicable to PD patients who were newly diagnosed and followed up in our single center and therefore does not reflect general PD patients, especially those in the prodromal, middle or late stage of PD. Nonetheless, the sample size for this study was large enough for analysis. Second, a previous study strongly suggested that a certain proportion of PD patients can develop different motor subtypes of PD after long periods of time. ${ }^{42}$ A longitudinal study should be conducted to verify our results. Third, we did not use a detailed instrument to validate the relationship between pain and the PIGD phenotype. In the future, it will be helpful to use more comprehensive methods (such as KPPS) to assess pain.

\section{Conclusion}

Patients with the PIGD subtype exhibited more severe cardiovascular symptoms, sleep impairments, and mood disturbances compared with those with the non-PIGD subtype, which could be largely attributed to an underlying NE deficiency in PIGD subtype patients. Furthermore, we demonstrated for the first time that pain is associated with the PIGD phenotype. Prompt detection and early treatment of these NMSs in PIGD patients may improve their treatment and management outcomes.

\section{Data Sharing Statement}

The authors declare that data in the manuscript are available from the corresponding author via email under reasonable requests.

\section{Acknowledgments}

We would like to thank the patients for their participation and their families for their support during the study.

\section{Funding}

This research was supported by 1) National Key Research and Development Program of China [grant NO. 2017YFC1310302, 2016YFC1306600], 2) General Program of National Natural Science Foundation of China [grant NO. 81571348], 3) Science and Technology Program of Jiangsu 
Province [grant NO. BE2019611], and 4) General Program of Jiangsu Provincial Natural Science Foundation of China [grant NO. BK20151077].

\section{Disclosure}

The authors declare no financial or other types of conflict of interest.

\section{References}

1. Kalia LV, Lang AE. Parkinson's disease. Lancet. 2015;386 (9996):896-912. doi:10.1016/s0140-6736(14)61393-3

2. Jankovic J, McDermott $M$, Carter $J$, et al. Variable expression of Parkinson's disease: A base-line analysis of the DATATOP cohort. Neurology. 1990;40(10):1529-1534. doi:10.1212/wnl.40.10.1529

3. Zetusky WJ, Jankovic J, Pirozzolo FJ. The heterogeneity of Parkinson's disease: clinical and prognostic implications. Neurology. 1985;35(4):522-526. doi:10.1212/wn1.35.4.522

4. Huber SJ, Paulson GW, Shuttleworth EC. Relationship of motor symptoms, intellectual impairment, and depression in Parkinson's disease. J Neurol Neurosurg Psychiatry. 1988;51(6):855-858. doi:10.1136/jnnp.51.6.855

5. Mortimer JA, Pirozzolo FJ, Hansch EC, Webster DD. Relationship of motor symptoms to intellectual deficits in Parkinson disease. Neurology. 1982;32(2):133-137. doi:10.1212/wnl.32.2.133

6. van der Heeden JF, Marinus J, Martinez-Martin P, RodriguezBlazquez C, Geraedts VJ, van Hilten JJ. Postural instability and gait are associated with severity and prognosis of Parkinson disease. Neurology. 2016;86(24):2243-2250. doi:10.1212/wnl.0000 000000002768

7. Pfeiffer RF. Non-motor symptoms in Parkinson's disease. Parkinsonism Relat Disord. 2016;22(Suppl 1):S119-122. doi:10.1016/j.parkreldis.2015.09.004

8. Mantri S, Morley JF, Siderowf AD. The importance of preclinical diagnostics in Parkinson disease. Parkinsonism Relat Disord. 2019;64:20-28. doi:10.1016/j.parkreldis.2018.09.011

9. Berg D, Postuma RB, Adler CH, et al. MDS research criteria for prodromal Parkinson's disease. Mov Disord. 2015;30(12):1600-1611. doi:10.1002/mds.26431

10. Heinzel S, Berg D, Gasser T, Chen H, Yao C, Postuma RB. Update of the MDS research criteria for prodromal Parkinson's disease. Mov Disord. 2019;34(10):1464-1470. doi:10.1002/mds.27802

11. Simuni T, Sethi K. Nonmotor manifestations of Parkinson's disease. Ann Neurol. 2009;64(Suppl S2):S65-80. doi:10.1002/ana.21472

12. Barone P, Antonini A, Colosimo C, et al. The PRIAMO study: A multicenter assessment of nonmotor symptoms and their impact on quality of life in Parkinson's disease. Mov Disord. 2009;24 (11):1641-1649. doi:10.1002/mds.22643

13. Schrag A. What contributes to quality of life in patients with Parkinson's disease? J Neurol Neurosurg Psychiatry. 2000;69 (3):308-312. doi:10.1136/jnnp.69.3.308

14. Todorova A, Jenner P, Ray Chaudhuri K. Non-motor Parkinson's: integral to motor Parkinson's, yet often neglected. Pract Neurol. 2014;14(5):310-322. doi:10.1136/practneurol-2013-000741

15. Huang X, Ng SY, Chia NS, et al. Non-motor symptoms in early Parkinson's disease with different motor subtypes and their associations with quality of life. Eur $J$ Neurol. 2019;26(3):400-406. doi:10.1111/ene. 13803

16. Deng X, Xiao B, Li -H-H, et al. Sexual dysfunction is associated with postural instability gait difficulty subtype of Parkinson's disease. $J \quad$ Neurol. 2015;262(11):2433-2439. doi:10.1007/s00415-015-7 55-8
17. Lichter DG, Benedict RHB, Hershey LA. Importance of BalanceGait Disorder as a Risk Factor for Cognitive Impairment, Dementia and Related Non-Motor Symptoms in Parkinson's Disease. J Parkinson Dis. 2018;8(4):539-552. doi:10.3233/jpd181375

18. Gibb WR, Lees AJ. The relevance of the Lewy body to the pathogenesis of idiopathic Parkinson's disease. J Neurol Neurosurg Psychiatry. 1988;51(6):745-752. doi:10.1136/jnnp.51.6.745

19. Chaudhuri KR, Martinez-Martin P, Schapira AHV, et al. International multicenter pilot study of the first comprehensive self-completed nonmotor symptoms questionnaire for Parkinson's disease: the NMSQuest study. Mov Disord. 2006;21(7):916-923. doi:10.1002/ mds. 20844

20. Chaudhuri KR, Martinez-Martin P, Brown RG, et al. The metric properties of a novel non-motor symptoms scale for Parkinson's disease: results from an international pilot study. Mov Disord. 2007;22(13):1901-1911. doi:10.1002/mds.21596

21. Konno T, Deutschländer A, Heckman MG, et al. Comparison of clinical features among Parkinson's disease subtypes: A large retrospective study in a single center. J Neurol Sci. 2018;386:39-45. doi:10.1016/j.jns.2018.01.013

22. Zhong LL, Song YQ, Cao H, Ju KJ, Yu L. The non-motor symptoms of Parkinson's disease of different motor types in early stage. Eur Rev Med Pharmacol Sci. 2017;21(24):5745-5750. doi:10.26355/eurrev_20171 2_14021

23. Simuni T, Caspell-Garcia C, Coffey C, Lasch S, Tanner C, Marek K. How stable are Parkinson's disease subtypes in de novo patients: analysis of the PPMI cohort? Parkinsonism Relat Disord. 2016;28:62-67. doi:10.1016/j.parkreldis.2016.04.027

24. Nutt JG. Motor subtype in Parkinson's disease: different disorders or different stages of disease? Mov Disord. 2016;31(7):957-961. doi: $10.1002 / \mathrm{mds} .26657$

25. The Parkinson Study Group. Effect of Deprenyl on the Progression of Disability in Early Parkinson's Disease. N Eng j Med. 1989;321 (20):1364-1371. doi:10.1056/nejm198911163212004

26. Espay AJ, LeWitt PA, Kaufmann H. Norepinephrine deficiency in Parkinson's disease: the case for noradrenergic enhancement. Mov Disord. 2014;29(14):1710-1719. doi:10.1002/mds.26048

27. Paulus W, Jellinger K. The Neuropathologic Basis of Different Clinical Subgroups of Parkinson's Disease. J Neuropathol Exp Neurol. 1991;50(6):743-755. doi:10.1097/00005072-19911100000006

28. Tohgi $\mathrm{H}$, Abe T, Takahashi $\mathrm{S}$, et al. Monoamine metabolism in the cerebrospinal fluid in Parkinson's disease: relationship to clinical symptoms and subsequent therapeutic outcomes. $J$ Neural Transm Park Dis Dement Sect. 1993;5(1):17-26. doi:10.1007/bf02260911

29. Samuels ER, Szabadi E. Functional neuroanatomy of the noradrenergic locus coeruleus: its roles in the regulation of arousal and autonomic function part I: principles of functional organisation. Curr Neuropharmacol. 2008;6(3):235-253. doi:10.2174/15701590878577 7229

30. Remy P, Doder M, Lees A, Turjanski N, Brooks D. Depression in Parkinson's disease: loss of dopamine and noradrenaline innervation in the limbic system. Brain. 2005;128(6):1314-1322. doi:10.1093/brain/ awh445

31. Hakusui S, Yasuda T, Yanagi T, et al. A radiological analysis of heart sympathetic functions with meta-[123I]iodobenzylguanidine in neurological patients with autonomic failure. J Auton Nerv Syst. 1994;49 (1):81-84. doi:10.1016/0165-1838(94)90023-x

32. Courbon F, Brefel-Courbon C, Thalamas C, et al. Cardiac MIBG scintigraphy is a sensitive tool for detecting cardiac sympathetic denervation in Parkinson's disease. Mov Disord. 2003;18 (8):890-897. doi:10.1002/mds.10461

33. Goldstein DS, Sharabi Y, Karp BI, et al. Cardiac sympathetic denervation preceding motor signs in Parkinson disease. Clin Autonomic Res. 2007;17(2):118-121. doi:10.1007/s10286-007-0396-1 
34. Borgonovo J, Allende-Castro C, Laliena A, Guerrero N, Silva H, Concha ML. Changes in neural circuitry associated with depression at pre-clinical, pre-motor and early motor phases of Parkinson's disease. Parkinsonism Relat Disord. 2017;35:17-24. doi:10.1016/j. parkreldis.2016.11.009

35. Schrag A, Horsfall L, Walters K, Noyce A, Petersen I. Prediagnostic presentations of Parkinson's disease in primary care: a case-control study. Lancet Neurol. 2015;14(1):57-64. doi:10.1016/s14744422(14)70287-X

36. Starkstein SE, Merello M, Jorge R, et al. A validation study of depressive syndromes in Parkinson's disease. Mov Disord. 2008;23 (4):538-546. doi:10.1002/mds. 21866

37. Nègre-Pagès L, Grandjean H, Lapeyre-Mestre M, et al. Anxious and depressive symptoms in Parkinson's disease: the French crosssectional DoPaMiP study. Mov Disord. 2010;25(2):157-166. doi: $10.1002 / \mathrm{mds} .22760$

38. Sung S, Vijiaratnam N, Chan DWC, Farrell M, Evans AH. Pain sensitivity in Parkinson's disease: systematic review and metaanalysis. Parkinsonism Relat Disord. 2018;48:17-27. doi:10.1016/j. parkreldis.2017.12.031
39. Lee JW, Song YS, Kim H, Ku BD, Lee WW. Alteration of Tremor Dominant and Postural Instability Gait Difficulty Subtypes During the Progression of Parkinson's Disease: analysis of the PPMI Cohort. Front Neurol. 2019;10:471. doi:10.3389/fneur.2019.00471

40. Rodríguez-Violante M, de Saráchaga AJ, Cervantes-Arriaga A, et al. Self-Perceived Pre-Motor Symptoms Load in Patients with Parkinson's Disease: A Retrospective Study. J Parkinson Dis. 2016;6(1):183-190. doi:10.3233/jpd-150705

41. Rodríguez-Violante M, Alvarado-Bolaños A, Cervantes-Arriaga A, Martinez-Martin P, Rizos A, Chaudhuri KR. Clinical Determinants of Parkinson's Disease-associated Pain Using the King's Parkinson's Disease Pain Scale. Mov Disord Clin Pract. 2017;4(4):545-551. doi: $10.1002 / \mathrm{mdc} 3.12469$

42. Eisinger RS, Hess CW, Martinez-Ramirez D, et al. Motor subtype changes in early Parkinson's disease. Parkinsonism Relat Disord. 2017;43:67-72. doi:10.1016/j.parkreldis.2017.07.018

\section{Publish your work in this journal}

Neuropsychiatric Disease and Treatment is an international, peerreviewed journal of clinical therapeutics and pharmacology focusing on concise rapid reporting of clinical or pre-clinical studies on a range of neuropsychiatric and neurological disorders. This journal is indexed on PubMed Central, the 'PsycINFO' database and CAS, and is the official journal of The International Neuropsychiatric Association (INA). The manuscript management system is completely online and includes a very quick and fair peer-review system, which is all easy to use. Visit http://www.dovepress.com/testimonials.php to read real quotes from published authors. 